\title{
Improvement of Solar Parabolic Concentrator Thermal Efficiency Correlated To Different Factors
}

\author{
Safa skouri ${ }^{1}$, Salwa bouadila ${ }^{1}$, Sassi Ben Nassrallah ${ }^{2}$ \\ I'The Research and Technology Center of Energy, The Thermal Processes Laboratory, Hammam Lif, B.P. 95, \\ 2050 Tunis, Tunisia) \\ ${ }^{2}$ (National Engineering School of Monastir, Street Ibn El Jazzar, 5019 Monastir, Tunisia)
}

\begin{abstract}
The purpose of this work is the characterization of a solar parabolic concentrator (SPC) designed, constructed and tested in the Research and Technologies Centre of Energy in Tunisia (CRTEn) after different modifications realized to the prototype in order to improve the performance of the system. In the first step a modification in the mechanical structure has been done and an optimization of the most performed reflector material has been done by an experimental study of three types of reflectors. In the second step a comparative study of three types of sun tracking systems with two axes is realized to choose the most one. Finally an experimental study is carried out to evaluate the solar parabolic concentrator thermal efficiency after the SPC mechanical and optical optimization. The thermal energy efficiency varies from 40\% to 77\%; the concentrating system reaches an average concentration factor around 178.
\end{abstract}

Keywords: Mechanical structure, reflector, sun tracking, thermal efficiency

\section{Introduction}

The idea of solar-energy concentration is not a new one. The early trials of using solar concentrators go back to the late years of the nineteenth century [1-4]. In the last decades an experimental investigation of a box type solar cooker employing a no tracking concentrator has been done by Negi and Purohit [5] they show that this device can provide improved heat collection and more efficient cooking. The cooker is approximately $10 \%$ costlier than the conventional box type solar cooker with a booster mirror. Sun-tracking system plays an important role in the development of high solar concentration applications that directly convert the solar energy into thermal or electrical energy. The thermal performance of the concentrating collectors is sensitive to the optical and geometric parameters. We can mention numerical and experimental studies of optical SPC parameters have been done to obtain the optimum thermal performance Zhiqiang et al. [6] presented a procedure to design a facet concentrator the optimum size and position of each facet are determined using Monte Carlo ray tracing analysis to achieve the most concentrated flux. Nepveu et al. [7] presented a global thermal model of the energy conversion of the $10 \mathrm{kWel}$ Eurodish dish/ Stirling unit erected at the CNRSPROMES laboratory in Odeillo. Using optical measurements made by DLR, they calculated the losses by parabola reflectivity and spillage. Many research works has been done by developing different types of sun tracking systems [8-10].

The aim of this work is to quantify the solar thermal efficiency of the SPC concentrator and after modifications.

\section{Description Of The New And The Former Transmission Mechanism}

The experimental rig designed and constructed is a solar parabolic concentrator SPC. The experimental system of the solar parabolic concentrator SPC is mainly composed of a parabola provided with an absorber placed by three arms at the focal position. A reflector embedded in a nacelle rotatable around two axes: the horizontal axis (elevation angle) from the support supported by a mast and the second is the vertical axis (azimuth angle) and counterbalancing. The experimental rig is fixed to the ground by a frame.

In order to ameliorate the SPC position accuracy, the previous mechanical structure was modified and the screw-nutactuators mechanical system was replaced by a new reduction gear unit (WG075-40-E-F) transmission mechanism. The new mechanism will permit two rotation movements with advanced mobility degree. The choice of the gear boxis related to its design. It is a worm gear speed reducer type which allows a significant rotational speed reduction (upto100) that increases its mechanical torque mean while the nonreversible aspect of the gear provides a position locking for the SPC. All the mentioned characteristics of the transmission mechanism will enhance the position stability and accuracy movement of the SPC. A low rotational speed combined with an important mechanical torque provides a better precision and a good stability of the sun tracking system. Each gear unites driven by a DC motor that puts the parabola mechanism in rotation around the two axes. 


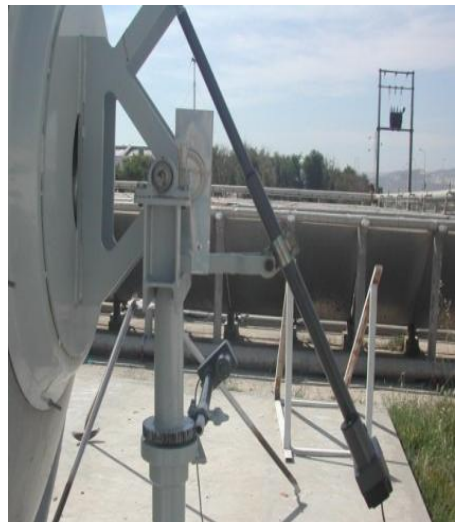

(a) Old transmission mechanism

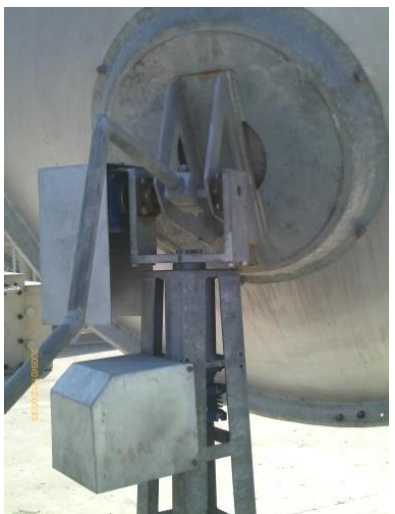

(b) New transmission mechanism

Fig.1. Transmission mechanism modification (a), (b)

\section{Comparative Study Of Three Types Of Sun Tracking Systems With Two Axes}

The tracking position of a mobile object has been the objective of several research studies in order to provide the adequate pursuit systems for specific applications. Concentrated solar technologies efficiency relies partially on solar tracking ability and the development of such systems become essential. However, this work contributed towards that goal, with a comparative experimental study of three different sun tracking systems used to ensure the movement of a solar parabolic concentrator around two axes.

The LDR sun tracking system the first sun tracking system is an azimuth-altitude dual axis tracker, which displaced the SPC reflector facing the sun by two screw-nutactuators. It is considered as an active tracking system. This tracker measures the sunlight intensity to determine its position.

First date and time based sun tracking system the date and time based sun tracking system is a programmed dual axes tracker. It is a passive sun tracking system (Fig. 8). The parabolic collector is oriented towards the sun with two direct current (DC) electric motors powered by electrical control unit basing on the signal delivered by a control module.

The second date and time based sun tracking system provided an advanced accuracy $(2.5 \mathrm{mrad})$ and a better stability with relatively cheap price $(1300 €)$. It is the adequate follower of this application. The proposed two-axis sun tracking system was characterized by movement stability and a fairly simple and low-cost electromechanical set-up with low maintenance requirements and ease of installation and operation.

Table 1: Cost estimation of different components of each sun tracking system

\begin{tabular}{|c|c|c|c|}
\hline Sun tracker & components & $\operatorname{Cost}(€)$ & Total cost \\
\hline \multirow[t]{4}{*}{ LDR sun tracking system } & Two Screw-nut actuators & 250 & \multirow{4}{*}{$\sim 1.000 €$} \\
\hline & Two LDR sensors & 100 & \\
\hline & PIC microcontroller 16F876, & 600 & \\
\hline & Two integrated circuit ULN 2003 & 50 & \\
\hline \multirow{3}{*}{$\begin{array}{l}\text { First date and time based } \\
\text { sun tracking system }\end{array}$} & Two Screw-nut actuators & 250 & \multirow{3}{*}{$\sim 2.000 €$} \\
\hline & Electrical control circuit ECC & 140 & \\
\hline & Agilent-3970 with HP control module & 1800 & \\
\hline \multirow{4}{*}{$\begin{array}{l}\text { Second date and time } \\
\text { based sun tracking } \\
\text { system }\end{array}$} & Two Reduction gear unit (WG075-40-E-F) & 300 & \multirow{4}{*}{$\sim 1300 €$} \\
\hline & Two DC motors & 100 & \\
\hline & Two electrical control circuit (ECC) & 300 & \\
\hline & Programmable logic controller (PLC) & 600 & \\
\hline
\end{tabular}

\section{Optimization of The SPC Reflector Material}

Fig. 2 gives the variation of the receiver temperature as function of time using three types of reflectors. These experiences are done in 3 days under comparative climatic conditions. An average insulation and ambient temperature equal to $800 \mathrm{~W} / \mathrm{m} 2$ and $25{ }^{\circ} \mathrm{C}$ respectively. The first reflector (R1) is aluminum sheet with reflecting coefficient of 0.80 ; we note that the obtained receiver temperature with this reflector is around $250{ }^{\circ} \mathrm{C}$. Using the second reflector (R2) that is aluminum paper with reflecting coefficient of 0.85 , the average absorber temperature is equal to $320{ }^{\circ} \mathrm{C}$. The last reflector (R3), 3M solar mirror film 1100 , which gives an absorber temperature around $900{ }^{\circ} \mathrm{C}$. Therefore, the variation average instantaneous temperature obtained using those reflectors, the most perform SPC reflector is the $3 \mathrm{M}$ solar mirror film 1100. That is a silver metalized weather able acrylic film. It has a solar weighted total hemispherical reflectance of $94 \%$ at air mass $1.5^{\circ}$. Solar mirror 
film offers a number of significant advantages over traditional glass mirrors, including higher reflectance, less weight and improved mechanical properties.

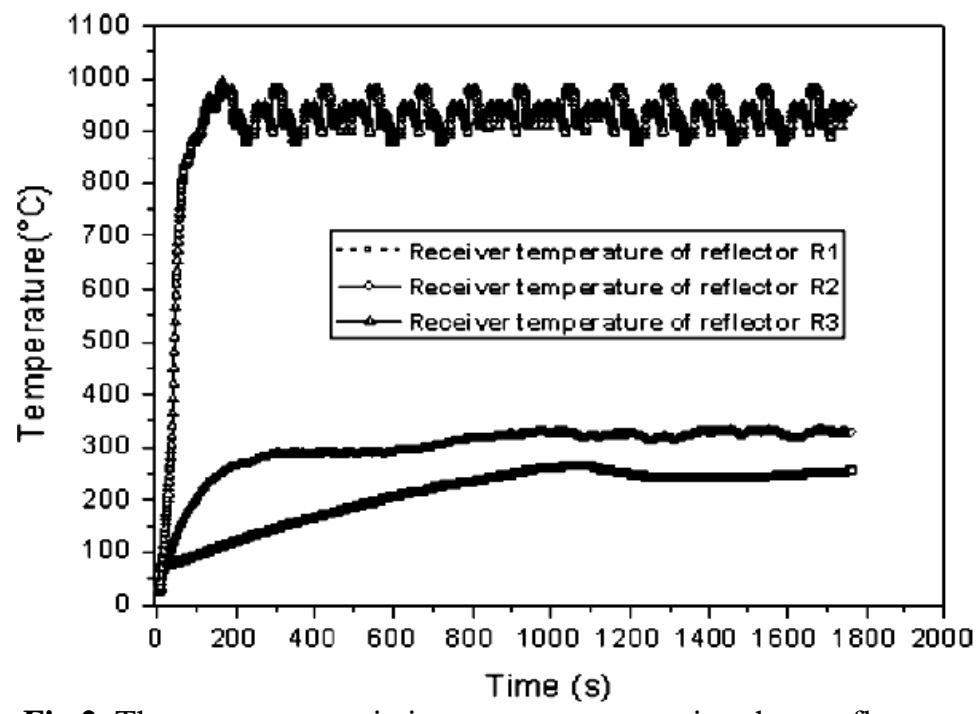

Fig.2. The temporary variations temperatures using three reflectors.

\section{Energy Analysis}

To determine the concentrated solar flux after all changes made at the parabolic solar concentrator. We used The disk method is based on the determination of concentrated flux using the energy balance by determining the different thermal losses .while 5 type $\mathrm{K}$ thermocouples (with $\pm 0.5^{\circ} \mathrm{C}$ accuracy) were used for instrumentation of the receiver disk. The latter were placed in the internal face of the plate at $\mathrm{z}=0.005 \mathrm{~m}$ depth. In Table 2 the radial locations of these thermocouples are reported, and in Fig. 3 their distribution is shown. Two thermocouples are used for each radial position. The thermocouples were channeled through grooves of $0.005 \mathrm{~m}$ depth,

Table 2 Characteristics of the disk.

\begin{tabular}{|c|c|c|}
\hline \multicolumn{3}{|c|}{ Material of the disk: stainless Diameter of the disk: $0.012 \mathrm{~m}$} \\
\hline Position in the disk & Symbol of the position & Distance from the center of the disk, $\mathrm{m}$ \\
\hline Position 1 & P1 & 0.05 \\
\hline Position 2 & P2 & 0.025 \\
\hline Position 3 & P3 & 0 \\
\hline Position 4 & P4 & 0.025 \\
\hline Position 5 & P5 & 0.05 \\
\hline
\end{tabular}

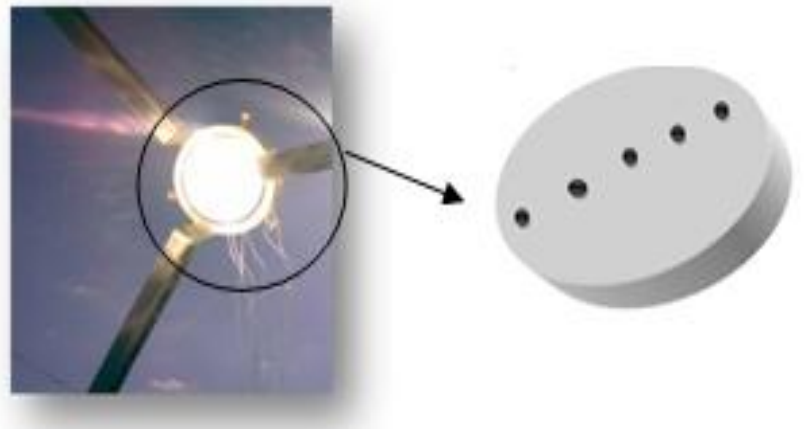

Fig. 3. Disk receiver.

The energy analysis is carried out to evaluate the system efficiency. The energy analysis presented in this section is mainly based on the first law of thermodynamics. The theoretical model employed for the study of the SPC is based on a thermal energy balance. The solar power absorbed $Q_{\mathrm{a}}$ by the receiver of solar parabolic dish system is given by [11]: 
$Q_{\mathrm{a}}=G^{*} A_{a} \eta_{o p}$

Where $G^{*}, A_{a}$ and $\eta_{o p}$ are solar beam radiation, aperture area of dish concentrator and optical efficiency, respectively. The following equation form can be used to perform an approximated optical efficiency analysis as.

$$
\eta_{o p}=\gamma \lambda \rho \tau \alpha
$$

Where $\lambda, \rho$ and $\tau \cdot \alpha$ are the factor of un-shading, dish reflectance and transmittance-absorptance product, respectively. $\gamma$ is the intercept factor of receiver, which is defined as the ratio of the energy intercepted by the receiver to the energy reflected by the focusing device

the useful thermal power gain $Q_{u}$ can be determined by.

$Q_{u}=Q_{a}-Q_{p}$

Where $Q_{\mathrm{a}}$ is the solar power absorbed by the receiver of solar parabolic dish system and $Q_{p}$ heat losses from a receiver occur due to the temperature difference between the receiver, conductive losses $Q_{p \cdot c d}$, convective losses $Q_{p \cdot c v}$ and radiative losses.

$$
Q_{p}=Q_{p \cdot c v}+Q_{p \cdot r}+Q_{p \cdot c d}
$$

Heat losses from a receiver $Q_{p}$ occur due to the temperature difference between the receiver and its surroundings and depend on the geometry of the receiver and the collector. In this study, heat losses from the receivers are classified as conductive loss $Q_{p \cdot c d}$, convective loss $Q_{p \cdot c v}$ and radiative loss by surface emission from the inner surface of the receiver $Q_{p \cdot r}$. Research has showed that the receiver conduction losses $Q_{p \cdot c d}$ represent a small fraction of the receiver thermal losses [11]. In order to evaluate the conductive loss from receiver, the following equation is used [11].

$$
Q_{p \cdot c d}=\left[\left(1 /\left(1 / A_{0} h\right)\right)+\left(l / K_{t}\left(A_{0} A_{w}\right)^{1 / 2}\right)\right] *\left(T_{r}-T_{a}\right)
$$

To determine the outside convective heat transfer coefficient by wind $h_{w}$, the following correlations recommended by McAdams [11] for the flow of air across a tube can be used.

$$
\left\{\begin{array}{lll}
h_{w} D_{0} / k_{\text {air }}=0.40+0.54\left(v_{w} D_{0} / v_{\text {air }}\right)^{0.52} & \text { for } & 0.1<v_{w} D_{0} / v_{\text {air }}<1000 \\
h_{w} D_{0} / k_{\text {air }}=0.30\left(v_{w} D_{0} / v_{\text {air }}\right)^{0.6} & \text { for } & 1000<v_{w} D_{0} / v_{\text {air }}<50000
\end{array}\right\}
$$

The convective losses in the receiver represent a significant fraction of the total losses in a dish system. Convective losses are a function of cavity temperature and geometry, aperture orientation, diameter, and wind velocity. Convection loss $Q_{p \cdot c v}$ of the receiver system can be calculated using the Newton's Cooling Law.

$$
Q_{p \cdot c v}=h_{c v} A_{c}\left(T_{r}-T_{a}\right)
$$

The radiation losses in the receiver contribute to a significant fraction of the total losses in the receiver and in the total dish system. Unlike convection losses; radiation losses are relatively constant throughout the day once a steady-state temperature has been reached for the heater head temperature. Radiation loss $Q_{\text {rad }}$ of the receiver system is given by:

$$
Q_{\text {rad }}=Q_{\text {rad.ref }}+Q_{\text {rad.emit }}
$$

The radiation losses due to reflectance $Q_{\text {rad.ref }}$ of the cavity surfaces depend of the cavity receiver effective absorptance, and it is given by the following equation:

$Q_{\text {rad.ref }}=\left(1-\alpha_{\text {eff }}\right) Q_{a}$

The effective absorptance is given by the following equation [11]:

Solar radiation is being reflected off from the cavity walls and back through the aperture. The general equation for net radiation exchange $[31,34]$ due to emission is given by the following equation:

$$
Q_{\text {rad.emit }}=\varepsilon_{\text {eff }} \sigma A_{c}\left(T_{r}^{4}-T_{a}^{4}\right)
$$


The thermal efficiency of the concentrator $\eta_{e n}$ based on the first law of thermodynamics is defined as the ratio between the useful energy and the direct solar radiation incident on the collector:

$\eta_{e n}=100 Q_{u} / I_{h}^{*}$

The mean concentration factor of the system is giving by:

$C_{R}=A_{c} Q_{u} / A_{a} I_{h}^{*}$

$I_{h}^{*}$ is The direct solar radiation

\section{Results And Discussion}

Once the analytical study has been fit to the experimental results, it is possible to evaluate temperatures at different positions inside the disk, and in particular, on the center of the disk (P3). In Fig.4 the temporary temperature distribution inside the disk is presented. As can be observed in this figure, the mean value of the temperature on the surface of the receiver plate (is around $650{ }^{\circ} \mathrm{C}$ ) is very high, and very different to the ambient temperature $\left(23^{\circ} \mathrm{C}\right)$.the most temperature obtained in the disk is in the center, we remark a symmetric temperature distribution .

Nevertheless, the heat losses by convection and radiation are small, due to the size of the spot and the mean temperature of the disk. In fig.10 are summarized the average heat losses from the disk in different positions we note that the convective heat losses towards the ambient are close to $15 \%$ and the radiative heat losses are close to $80 \%$.it is clear that conduction losses is neglected in thermal balance and radiation losses are so important in concentration technologies. We show that minimum overall losses are in position 3 at the center of the disk.

Experimental results of the energetic efficiency of the solar parabolic concentrator after and before modification using the disk as an absorber are depicted in Fig.6. it reach her maximum at the center of the disk that is around $15 \%$ using the previous prototype and after mechanical, optical and tracking modifications we obtained a performed solar concentrator with average value of the thermal efficiency equal to $70 \%$.

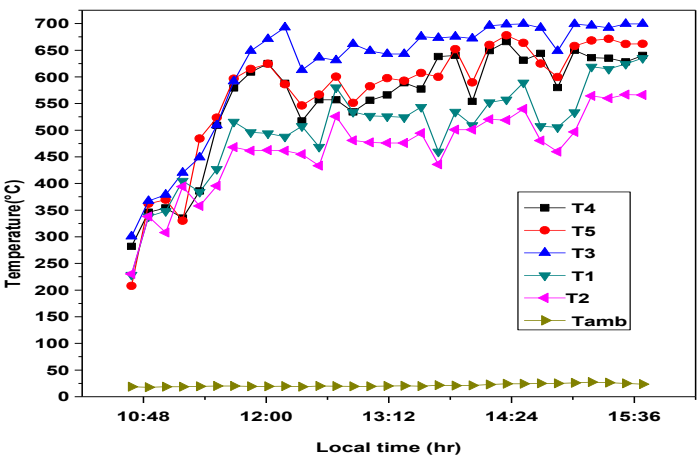

Fig. 4. Temporary temperature variation in different disk positions.

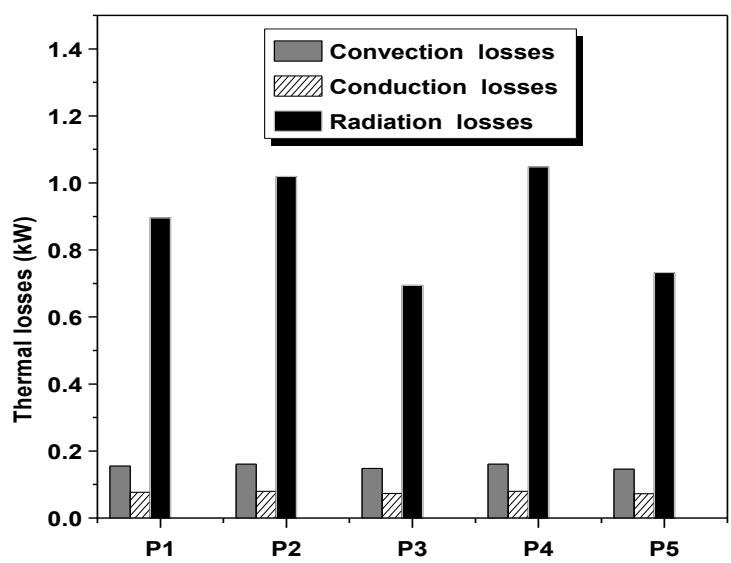

Fig. 5. Spatial variation of the SPC thermal losses. 


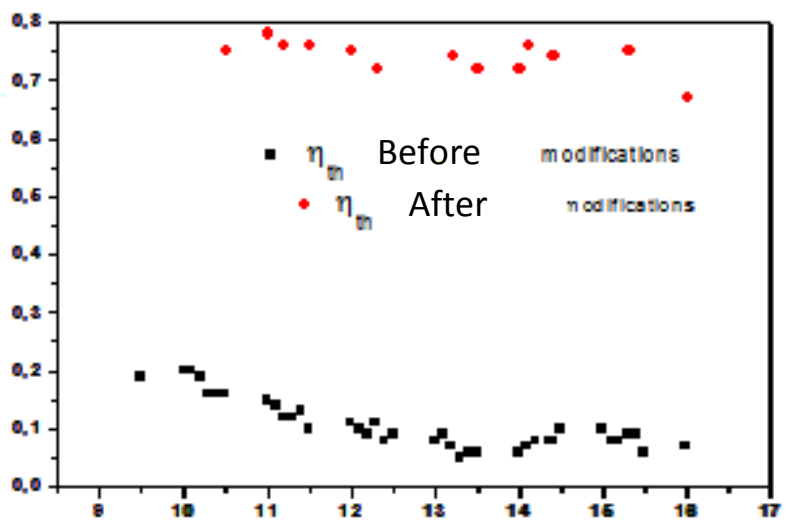

Fig. 6. Thermal efficiency of SPC after and before modifications

VII. Conclusion

This work is a contribution of a solar parabolic concentrator equipped with a programmed sun tracking system, an absorber and measuring instruments. The main findings of the present study to increase the thermal efficiency of the system is that after mechanical, optical and tracking modifications we obtained a performed solar concentrator with average value of the thermal efficiency equal to $70 \%$.

\section{NOMENCLATURE}

$A_{a} \quad$ Aperture area of dish concentrator, $m^{2}$.

$A_{0} \quad$ Area of the outer surface of the receiver, $m^{2}$.

$A_{c a} \quad$ Aperture area of the cavity, $m^{2}$.

$A_{c} \quad$ Total inner surface area of the cavity, $m^{2}$.

$A_{w} \quad$ Area of the inner side of receiver, $m^{2}$.

$C_{R} \quad$ Mean concentration factor.

$D_{0} \quad$ Dish diameter, $m$.

$I_{h}^{*} \quad$ Direct radiation, $W$.

$Q_{\text {rad.enit }} \quad$ Radiation losses due to emission, $W$.

$Q_{u} \quad$ Useful thermal power gain, $W$.

$Q_{p \cdot c v} \quad$ Convection losses, $W$.

$Q_{p \cdot c d} \quad$ Receiver conduction losses, $W$.

$Q_{p \cdot r} \quad$ Radiation losses, $W$.

$Q_{p} \quad$ Receiver thermal losses, $W$.

$Q_{\text {rad }, \text { ref }} \quad$ Radiation losses due to reflectance, $W$.

$Q_{a} \quad$ Solar power absorbed, $W$.

$G^{*} \quad$ Solar beam radiation, $W$.

$D^{*} \quad$ Solar diffuse radiation, $W$.

$T_{r} \quad$ Temperature of receiver, ${ }^{\circ} \mathrm{C}$.

$T_{a} \quad$ Ambient temperature, ${ }^{\circ} \mathrm{C}$. 


$\begin{array}{ll}\eta_{e n} & \text { Energy efficiency of SPC, } \% \\ \eta_{e x} & \text { Exergetic efficiency of SPC, \% } \\ \eta_{o p} & \text { Optical efficiency, } \% \\ \rho & \text { Dish reflectance } \\ \eta_{e n} & \text { Energy efficiency of SPC, } \% \\ \tau \alpha & \text { Transmittance-absorptance product. } \\ \gamma & \text { Intercept factor of receiver } \\ v_{\text {air }} & \text { Air viscosity, } m^{2} / s .\end{array}$

\section{References}

[1]. El-Refaie MF. Concentrated solar thermal power. Renew Energy Energy Efficiency MENA Region 2009.

[2]. Cleveland Cutler J, Frank S. Encyclopedia Earth 2008.

[3]. Thomas A. Solar steam generating systems using parabolic trough concentrators. Energy Convers Manage 1996;37:215-45.

[4]. Kreith F, Gowami DY. Handbook of energy efficiency and renewable energy. London, New York: Taylor and Francis Group; 2007.

[5]. Negi BS, Purohit I. Experimental investigation of a box type solar cooker employing a non-tracking concentrator. Energy Convers Manage 2005;46:577-604.

[6]. Zhiqiang L, Lapp J, Lipinski W. Optical design of a flat-facet solar concentrator. Sol Eng 2012;86:1962-6.

[7]. Nepveu F, Ferriere A, Bataille F. Thermal model of dish/Stirling systems. Sol Energy 2009;83:81-9.

[8]. Cruz-Peragón F, Pedro J, Peláez C, Francisco A, López-García R, Palomar JM. Anapproach to evaluate the energy advantage of two axes solar tracking systemsin Spain. Appl Energy 2011;88:5131-42.

[9]. Abdallah S, Badran OO. Sun tracking system for productivity enhancement of solar still. Desalination 2008;220:66976.

[10]. Sungur C. Multi-axes sun-tracking system with PLC control for photovoltaic panels in Turkey. Renew Energy 2009;34:1119-25.

[11]. Safa Skouri, Salwa Bouadila, Mohieddine Ben Salah, Sassi Ben Nasrallah Comparative study of different means of concentrated solar flux measurement of solar parabolic dish Energy Conversion and Management 76 (2013) 10431052 